\title{
Synthesis of regioselectively acylated quercetin analogues with improved antiplatelet activity
}

\author{
YU DUAN ${ }^{1,3^{*}}$, NA SUN $^{1}$, MIN XUE $^{1}$, XIAOLAN WANG ${ }^{1}$ and HU YANG $^{2-4 *}$ \\ ${ }^{1}$ School of Pharmacy, Weifang Medical College, Weifang, Shandong 261053, P.R. China; ${ }^{2}$ Department of Chemical \\ and Life Science Engineering, Virginia Commonwealth University, Richmond, VA 23119; ${ }^{3}$ Department of Pharmaceutics \\ and ${ }^{4}$ Massey Cancer Center, Virginia Commonwealth University, Richmond, VA 23298, USA
}

Received March 17, 2017; Accepted August 17, 2017

DOI: $10.3892 / \mathrm{mmr} .2017 .7781$

\begin{abstract}
The aim of the present study was to report on a complete synthetic approach, namely benzylation-hydrolysis-acylation-hydrogenation, to the synthesis of regioselectively acylated quercetin analogues using low-cost rutin as a starting material. Three quercetin analogues, quercetin-3-O-propionate (Q-pr), quercetin-3-O-butyrate (Q-bu) and quercetin-3-O-valerate (Q-va), containing 3-, 4- and 5-carbon aliphatic acyl chains, respectively, were synthesized and characterized with ${ }^{1} \mathrm{H}$ nuclear magnetic resonance (NMR), ${ }^{13} \mathrm{C}$ NMR and mass spectrometry. Compared with quercetin, all three analogues exhibited improved lipophilicity. The lipophilicity of the analogue increased with increasing acyl chain length. Q-va exhibited the highest lipophilicity among the three analogues, but a lower water solubility compared with quercetin. By contrast, Q-pr and Q-bu exhibited 8.2- and 4.7-fold higher water solubility compared with quercetin, respectively. The in vitro and in vivo studies demonstrated that Q-pr and Q-bu were more effective whereas Q-va was less effective in inhibiting platelet aggregation compared with quercetin. These results indicated that the water solubility and the lipophilicity of the analogues must be improved in order to achieve higher antiplatelet activity, and an optimal acyl chain length is crucial for the synthesized quercetin analogues to be more effective.
\end{abstract}

Correspondence to: $\mathrm{Dr} \mathrm{Yu}$ Duan, School of Pharmacy, Weifang Medical College, 7166 West Baotong Road, Weifang, Shandong 261053, P.R. China

E-mail: duanyu@wfmc.edu.cn

Dr Hu Yang, Department of Chemical and Life Science Engineering, Virginia Commonwealth University, 401 West Main Street, Richmond, VA 23119, USA

E-mail: hyang2@vcu.edu

\section{${ }^{*}$ Contributed equally}

Key words: quercetin, regioselective acylation, water solubility, oil-water partition coefficient, antiplatelet aggregation

\section{Introduction}

Quercetin is the most abundant dietary flavonoid (1). It was previously demonstrated that quercetin exerts an antiplatelet effect that may be utilized to prevent and alleviate cardiovascular diseases (2-8). However, quercetin exhibits low hydrophilicity and low water solubility. In particular, the low water solubility is associated with the limited bioavailability of quercetin, and remains as a major obstacle to its therapeutic applications $(9,10)$. The strong intermolecular packing of planar phenyl and hereto rings among quercetin molecules is presumably attributed to the low solubility of quercetin in water. Acylation of the hydroxyl groups of quercetin by an acyl donor containing a short aliphatic chain is a feasible way for destroying this intermolecular packing, hence improving the solvation of quercetin. However, non-selective acylation of hydroxyl groups may lead to loss of the biological activities of quercetin. The two adjacent hydroxyl groups at C3 and $\mathrm{C} 4$ in ring $\mathrm{B}$, and the double bond between $\mathrm{C} 2-\mathrm{C} 3$ and the carbonyl group at $\mathrm{C} 4$ in ring $\mathrm{C}$, are the most critical elements for quercetin to be biologically active $(11,12)$. Therefore, to improve quercetin water solubility without compromising its biological activities, these functional groups must be kept intact.

Regioselective acylation of the hydroxyl group at C3 offers a reasonable solution $(13,14)$. Chemo-enzymatic synthesis of regioselectively acylated quercetin has been previously investigated (13-17). However, synthesis methods without using enzymes are desirable, as they may reduce the reaction time and eliminate the need for costly enzymes. Only few studies using enzyme-free synthesis methods for the synthesis of quercetin analogues have been reported to date (18). A major challenge is that quercetin's hydroxyl groups may be randomly acylated, yielding a mixture of products. The aim of the present study was to develop a complete synthetic method (benzylation-hydrolysis-acylation-hydrogenation) for the synthesis of quercetin analogues using low-cost rutin as a starting material. With this method, regioselective acylation of C3 becomes possible as the hydroxyl of C3 is naturally protected with glycoside via a glycosidic bond. Three quercetin analogues were synthesized, quercetin-3-O-propionate (Q-pr), quercetin-3-O-butyrate $(\mathrm{Q}-\mathrm{bu})$ and quercetin-3-O-valerate (Q-va), with high yields (Fig. 1), and their water solubility, 
lipophilicity and antiplatelet activity were examined against quercetin.

\section{Materials and methods}

Chemicals. Rutin $(\geq 98 \%)$ was obtained from Nanjing TCM Institute of Chinese Materia Medica (Nanjing, China) and kept at $110^{\circ} \mathrm{C}$ under $1.3 \mathrm{kPa}$ for $12 \mathrm{~h}$ to remove bound water. Propionyl chloride, butyryl chloride and valeroyl chloride (analytical grade) were purchased from Aladdin Industrial Corporation (Shanghai, China). Benzyl bromide was obtained from Sinopharm Chemical Reagent (Shanghai, China) and distilled prior to use. $N, N$-dimethylformamide (DMF), triethylamine (TEA), and dichloromethane (DCM) were purchased form Tianjin Kemiou Chemical Reagent (Tianjin, China) and dehydrated prior to use. Adenosine diphosphate (ADP), arachidonic acid (AA) and platelet-activating factor (PAF) were purchased from Sigma-Aldrich (Merck KGaA, Darmstadt, Germany). Palladium on carbon $(\mathrm{Pd} / \mathrm{C})(10 \%)$ was obtained from Sinopharm Chemical Reagent.

Animals. Male New Zealand rabbits (license no. 0017075) and male Wistar rats (250-300 g; license no. 0010061) were obtained from the Experimental Animal Center of Shandong Lukang Pharmaceutical (Jinan, China). The experimental procedures were approved by the Animal Experimentation Ethics Committee of Weifang Medical College and conducted in accordance with the guidelines of the National Health and Medical Research Council of China for the care and use of animals.

Synthesis of acylated quercetin analogues. The synthesis followed a complete chemical synthetic procedure, namely benzylation-hydrolysis-acylation-hydrogenation, using rutin as the starting material.

Step 1. Rutin (24.42 g, $40 \mathrm{mmol})$ and $\mathrm{K}_{2} \mathrm{CO}_{3}(18.35 \mathrm{~g}$, $133 \mathrm{mmol}$ ) were sequentially added to $160 \mathrm{ml}$ of DMF under nitrogen and stirred for $1 \mathrm{~h}$ at room temperature (19). Subsequently, benzyl bromide ( $16 \mathrm{ml}, 133 \mathrm{mmol})$ was added dropwise into the reaction mixture. The reaction mixture was stirred at $40^{\circ} \mathrm{C}$ for $3 \mathrm{~h}$ under nitrogen, and then adjusted to $\mathrm{pH}$ 6.0 with $10 \%(\mathrm{v} / \mathrm{v})$ acetic acid in ice bath. Deionized water $(300 \mathrm{ml})$ was then added to this mixture and the suspension was filtered.

Step 2. The filtered residue was dissolved in $600 \mathrm{ml}$ of $95 \%(\mathrm{v} / \mathrm{v})$ ethanol at $70^{\circ} \mathrm{C}$. Hydrochloric acid $[90 \mathrm{ml}, 36 \%$ $(\mathrm{w} / \mathrm{w})]$ was added to the solution and the hydrolysis continued at $70^{\circ} \mathrm{C}$ for $2 \mathrm{~h}$. The suspension was cooled down to room temperature and then filtered to yield hydrolyzate. The hydrolyzate was washed with ice water until its $\mathrm{pH}$ became neutral.

Step 3. The hydrolyzate was dissolved in $250 \mathrm{ml}$ of DCM followed by addition of chloride (propionyl chloride, butyryl chloride or valeroyl chloride) (44 mmol) and TEA (44 mmol). The mixture was stirred at room temperature until thin-layer chromatography (TLC) analysis showed the completeness of the reaction. The reaction mixture was then extracted with $1 \mathrm{~mol} / \mathrm{l}$ hydrochloric acid, and the organic layer was washed with a saturated aqueous solution of $\mathrm{NaHCO}_{3}$ and deionized water. The pooled extracts were dried over $\mathrm{Na}_{2} \mathrm{SO}_{4}$ and

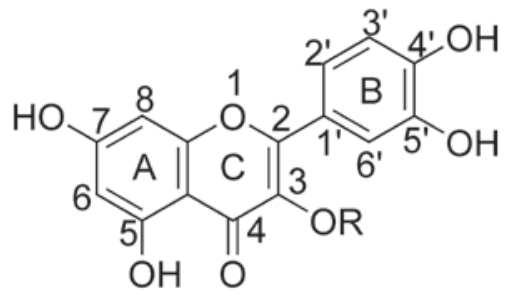

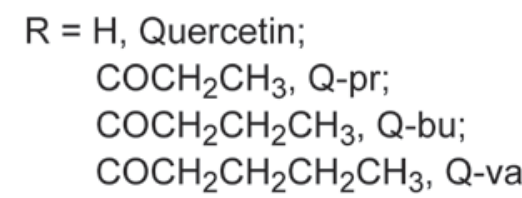

Figure 1. Chemical structures of quercetin and the synthesized quercetin analogues. Q-pr, quercetin-3- $O$-propionate; Q-bu, quercetin-3- $O$-butyrate; $\mathrm{Q}$-va, quercetin-3- $O$-valerate.

then taken to dryness under vacuum to retrieve the acylated compound.

Step 4. The acylated compound was dissolved in $5,000 \mathrm{ml}$ of ethanol/dioxane (1:1, v/v). Pd/C (2 g) was added to the solution. The reaction mixture was stirred at room temperature under hydrogen at atmospheric pressure for $2 \mathrm{~h}$. Pd/C was filtered off, and the filtrate was evaporated in vacuum to retrieve the crude product of acylated quercetin analogues.

Purification of acylated quercetin analogues. Acylated derivatives were purified by using semi-preparative high-performance liquid chromatography (HPLC). An Agilent 1200 infinity preparative chromatography system performed chromatographic separation; this system was equipped with an Agilent Prep LC controller, an ultraviolet (UV) detector (VWD-G1314B), a preparative column (ZORBAX-SB C18, 250x9.4 mm, $5 \mu \mathrm{m}$ ), an injector (3725i) and an auto-fraction collector (G1364C) (all from Agilent Technologies, Inc., Santa Clara, USA). Q-pr and Q-bu were purified using the linear elutions of acetonitrile/water (40:60, $\mathrm{v} / \mathrm{v}$ and 30:70, v/v, respectively). Q-va was purified using a linear gradient of acetonitrile and water for $0 \mathrm{~min}(30: 70, \mathrm{v} / \mathrm{v})$, $7 \min (40: 60, v / v), 15 \min (50: 50, v / v)$, and $25 \min (30: 70, v / v)$. The flow rate was $5 \mathrm{ml} / \mathrm{min}$, and the elution was performed at room temperature at $254 \mathrm{~nm}$ with the UV detector.

$T L C$. The acylation process was monitored using TLC on silica gel $60-\mathrm{GF}_{254}$ (Merck KGaA), with a solvent mixture of ethyl acetate, methanol and acetic acid (at a ratio of 6:4:0.1, $\mathrm{v} / \mathrm{v} / \mathrm{v})$. The plate was observed and detected under UV light $(254 \mathrm{~nm})$.

Nuclear magnetic resonance (NMR) spectroscopy and mass spectrometry $(M S)$. Quercetin and three acylated derivatives, Q-pr, Q-bu and Q-va, were characterized with ${ }^{1} \mathrm{H}$ NMR $(500 \mathrm{MHz})$ and ${ }^{13} \mathrm{C}$ NMR $(100 \mathrm{MHz})$ in DMSO- $d_{6}$ using a Bruker AV500 NMR spectrometer (Bruker Corporation, Billerica, MA, USA) with tetramethylsilane as an internal reference, and with MS without breaking into fragment ions using an Agilent 6410 liquid chromatography-mass spectrometer (Agilent Technologies, Inc.) with electrospray ionization 
Water solubility measurements. The water solubility of quercetin and the three acylated derivatives was determined according to the method described by Bonina et al (20). An excess amount of the compound was weighed into a glass tube containing $2 \mathrm{ml}$ of water, and the tube was sealed with a Teflon-lined cap. The mixture was stirred by a magnetic stirrer for $24 \mathrm{~h}$ at room temperature and was then filtered using a Millex HV13 filter unit $(0.22 \mu \mathrm{m}$; Merck KGaA). The drug concentration in the saturated solution at $254 \mathrm{~nm}$ was determined using a Shimadzu chromatography system (LC-20AT), equipped with a UV detector (SPD-M10Avp) and a column (Shim-pack VP-ODS C18, 250x4.60 mm, $5 \mu \mathrm{m}$ ) (all from Shimadzu, Kyoto, Japan). The mobile phase was water/methanol $(70: 30, \mathrm{v} / \mathrm{v})$ at a flow rate of $1 \mathrm{ml} / \mathrm{min}$.

Lipophilicity measurements. Lipophilicity may be estimated using reverse-phase chromatographic retention time due to the good association between the logarithm of the $n$-octanol/water partition coefficient $(\log P)$ and the $\log$ arithm of the capacity factor eluting with $100 \%$ water $\left(\log k_{\mathrm{w}}\right)$ determined using octadecyl silica columns. The value of $\log k_{\mathrm{w}}$ was obtained according to the method described by Braumann (21). Each compound was dissolved in methanol to a final concentration of $10 \mu \mathrm{g} / \mathrm{ml}$. The sample $(\mathrm{n}=3)$ was filtered prior to injection using a filter (Millex HV13, $0.22 \mu \mathrm{m}$; Merck KGaA) and the aliquot $(20 \mu \mathrm{l})$ was injected into the HPLC. The value of logarithm of the capacity factor $\left(\log k^{\prime}\right)$ was calculated using the following equation:

$$
\log k^{\prime}=\frac{t_{r}-t_{0}}{t_{0}}
$$

where $t_{r}$ is the retention time of the flavonoid peak and $t_{0}$ denotes the retention time of the non-retained solvent peak. The HPLC condition was maintained the same as in the water solubility test. Only the volume fraction of methanol in mobile phase was changed for each measurement. A series of values of retention times and $\log k^{\prime}$ were obtained. At a plot of $\log$ $k^{\prime}$ vs. the volume fraction of methanol in mobile phase, $\varphi$, extrapolated the data to $100 \%$ water to obtain the intersection point with the $y$ axis, that is $\log k_{\mathrm{w}}$ value. A formula (21), which exhibited an excellent correlation between $\log k_{\mathrm{w}}$ and $\log P$ for the compound containing conjugated aromatic, was selected to calculated the value of $\log P$ :

$$
\log k_{w}=0.988 \times \log P+0.020
$$

The lipophilicity of quercetin and the three acylated derivatives was also calculated using the ACD/ChemSketch (Advanced Chemistry Development Inc., Toronto, Canada) and the Property Explorer Applet (www. openmolecules. org).

The association between $\log k^{\prime}$ and $\varphi$, the values of $\log k_{\mathrm{w}}$ and $\log P$ of quercetin and the three acylated derivatives are presented in Table I.

In vitro antiplatelet activity test. Fresh whole blood was collected from healthy male New Zealand rabbits with 3.8\% $(\mathrm{w} / \mathrm{v})$ sodium citrate at a volume ratio of 9:1. Platelet-rich plasma (PRP) was obtained by centrifugation at $328.7 \mathrm{x}$ g for $8 \mathrm{~min}$ at room temperature with no brake. PRP $(300 \mu \mathrm{l})$ was incubated for $3 \mathrm{~min}$ at $37^{\circ} \mathrm{C}$ in the presence of various final concentrations $(0-350 \mu \mathrm{M})$ of quercetin or acylated analogue $(\mathrm{n}=8-10)$, predissolved in DMSO. The final concentration of DMSO was $<1.0 \%(\mathrm{v} / \mathrm{v})$ to eliminate false positive results. Subsequently, platelet aggregation was stimulated by ADP (7 $\mu \mathrm{M})$, AA $(350 \mu \mathrm{M})$, or PAF $(7.2 \mathrm{nM})$. The aggregation was monitored for $5 \mathrm{~min}$ using a platelet aggregometer (LBY-NJ4A, Precill, Beijing, China) with constant stirring at $241.5 \mathrm{x} \mathrm{g}$, and the aggregation rates were recorded to determine the percentage of aggregation.

In vivo antiplatelet activity test. Administration of investigational agents and sample preparation for platelet aggregation were performed according to the method described by Mosawy et al (22). In brief, the rats (8-10 per group) were treated with $0.03 \mathrm{mmol} / \mathrm{kg}$ quercetin, Q-pr, Q-bu, Q-va, or control consisting of $0.5 \%$ DMSO with $2.2 \mathrm{mM}$ polyethylene glycol (PEG) in saline. DMSO and PEG were used to improve the solubility of the investigational agents in the blood. The investigational agents were administered via a single intravenous (IV) bolus via the tail vein. Experimental procedures and blood sample collection were performed $30 \mathrm{~min}$ after the IV bolus treatment. In each rat, $5 \mathrm{ml}$ of fresh whole blood was collected into tubes containing $600 \mu \mathrm{l}$ of $3.8 \%$ (w/v) sodium citrate via cardiac puncture. PRP was obtained by centrifugation at $377.3 \mathrm{x} \mathrm{g}$ for $8 \mathrm{~min}$ at room temperature with no brake. PRP was incubated for $3 \mathrm{~min}$ at $37^{\circ} \mathrm{C}$, and platelet aggregation was then stimulated by $7 \mu \mathrm{M}$ ADP, $350 \mu \mathrm{M}$ AA, or $7.2 \mathrm{nM}$ PAF (final concentration). The aggregation was monitored for 5 min by using an aggregometer (LBY-NJ4A, Precill) at a constant stirring of $241.5 \mathrm{x} \mathrm{g}$, and the aggregation rates were recorded to determine the percent of aggregation. The value of the aggregation inhibitory rate (AIR) was calculated as follows:

$$
A I R \%=\left(1-\frac{A}{B}\right) \times 100 \%
$$

where $A$ is the platelet aggregation rate of the sample and $B$ is the platelet aggregation rate of the no compound treated group (control).

Statistical analysis. All values are expressed as mean \pm standard error of the mean. Comparisons between test samples and blank were performed using SPSS 9.0 software for Windows (SPSS Inc., Chicago, IL, USA) with Dunnett's test for post hoc comparisons, and $\mathrm{P} \leq 0.01$ was considered to indicate statistically significant differences.

\section{Results and Discussion}

The synthesis of the three quercetin analogues involves four steps (Fig. 2). The hydroxyl group at C5 is considered non-reactive, as it forms an intramolecular hydrogen bond with the carbonyl at C4 (22). Therefore, this hydroxyl group remained intact throughout the reactions. In step 1, anhydrous $\mathrm{K}_{2} \mathrm{CO}_{3}$ was added to facilitate the formation of oxygen anions for hydroxyl groups at C3, C4 and C7 of rutin. Subsequently, benzyl bromide was added to benzylate the forming oxygen anions. A protic solvent, DMF, was used to avoid the production of C-alkylated derivatives. The hydroxyl group at C3 was recovered after hydrolysis of the glycoside (step 2), and 
Table I. Association between $\log k^{\prime}$ and $\varphi$, and values of $\log k_{\mathrm{w}}, \log P$ and water solubility of quercetin, Q-pr, Q-bu and Q-va.

\begin{tabular}{llccccc}
\hline & & & \multicolumn{3}{c}{$\log P^{\mathrm{b}}$} & \\
Compound & $\log k^{\prime}-\varphi\left(\mathrm{R}^{\mathrm{a}}\right)$ & $\log k_{w}$ & $\mathrm{~A}$ & $\mathrm{~B}$ & $\mathrm{C}$ & Water solubility $(\mu \mathrm{g} / \mathrm{ml})$ \\
\hline Quercetin & $\log k^{\prime}=1.67-2.16 \varphi(\mathrm{R}=-0.98221)$ & 1.67 & 1.67 & 2.07 & 1.49 & 1.98 \\
Q-pr & $\log k^{\prime}=3.04-4.21 \varphi(\mathrm{R}=-0.9837)$ & 3.04 & 3.06 & 2.77 & 2.43 & 16.27 \\
Q-bu & $\log k^{\prime}=3.47-4.41 \varphi(\mathrm{R}=-0.9943)$ & 3.47 & 3.48 & 3.30 & 2.88 & 9.36 \\
Q-va & $\log k^{\prime}=3.72-4.77 \varphi(\mathrm{R}=-0.9995)$ & 3.72 & 3.75 & 3.83 & 3.34 & 1.07
\end{tabular}

${ }^{a}$ Linear correlation coefficient. ${ }^{b} \log P$ was calculated as follows: A, according to the formula $\log k_{w}=0.988-\log P_{O C T}+0.020$; B, using the $\mathrm{ACD} /$ ChemSketch; $\mathrm{C}$, using the Property Explorer Applet (www.openmolecules.org). Q-pr, quercetin-3-O-propionate; Q-bu, quercetin-3-O-butyrate; Q-va, quercetin-3- $O$-valerate.

became the only reactive group for subsequent acylation in step 3. In the final step, the benzyl groups at C3, C4 and C7 were removed by hydrogenation. The resulting acylated quercetin derivatives were purified using semi-preparative HPLC. All these derivatives were yellow powders.

The ${ }^{1} \mathrm{H}$ NMR, ${ }^{13} \mathrm{C}$ NMR and MS spectra collectively confirmed the structures of regioselectively acylated quercetin analogues.

$Q-p r .{ }^{1} \mathrm{H}$ NMR chemical shifts: $\delta$ (ppm) $12.23(1 \mathrm{H}, \mathrm{s}, 5-\mathrm{OH})$, 10.67 (1H, br, s, 7-OH), 9.72 (3H, br s, 3, 3', 4'-OH), 7.33 (1H, d, H-2') 7.27 (1H, d, H-6'), 6.92 (1H, d, H-5'), 6.48 (1H, d, H-8), 6.25 (1H, d, H-6), $2.68\left(2 \mathrm{H}, \mathrm{t}, \mathrm{CH}_{2}\right), 1.15\left(3 \mathrm{H}, \mathrm{t}, \mathrm{CH}_{3}\right) .{ }^{13} \mathrm{C} \mathrm{NMR}$ chemical shifts: $\delta$ (ppm) $175.39(\mathrm{C}-4), 171.78(\mathrm{C}=\mathrm{O}), 165.18$ (C-7), 161.54 (C-9), 157.08 (C-5), 156.36 (C-2), 149.78 (C-4'), 145.95 (C-3'), 130.06 (C-3), 120.98 (C-1'), 120.08 (C-6'), 116.45 (C-5'), 115.46 (C-2'), 103.89 (C-10), 99.55 (C-6), 94.56 (C-8), $27.08\left(\mathrm{CH}_{2}\right), 9.32\left(\mathrm{CH}_{3}\right)$. MS (m/z) for Q-pr: $381.1[\mathrm{M}+\mathrm{Na}]^{+}$.

$Q$-bu. ${ }^{1} \mathrm{H}$ NMR chemical shifts: $\delta$ (ppm) $12.20(1 \mathrm{H}, \mathrm{s}$, 5-OH), 7.32 (1H, d, H-2'), 7.26 (1H, d, H-6'), 6.91 (1H, d, H-5'), $6.47(1 \mathrm{H}, \mathrm{d}, \mathrm{H}-8), 6.25$ (1H, d, H-6), $2.63\left(2 \mathrm{H}, \mathrm{t}, \mathrm{CH}_{2}\right), 1.66(2 \mathrm{H}$, $\left.\mathrm{m}, \mathrm{CH}_{2}\right), 0.96\left(3 \mathrm{H}, \mathrm{t}, \mathrm{CH}_{3}\right) .{ }^{13} \mathrm{C} \mathrm{NMR}$ chemical shifts: $\delta(\mathrm{ppm})$ 174.95 (C-4), 170.48 (C=O), 164.72 (C-7), 161.08 (C-9), 156.63 (C-5), 156.01 (C-2), 149.31 (C-4'), 145.45 (C-3'), 129.56 (C-3), 120.54 (C-1'), 119.60 (C-6'), 115.95 (C-5'), 115.01 (C-2'), 103.55 (C-10), 99.11 (C-6), $94.12(\mathrm{C}-8), 28.85\left(\mathrm{CH}_{2}\right), 17.88\left(\mathrm{CH}_{2}\right)$, $13.33\left(\mathrm{CH}_{3}\right)$. MS (m/z): $395.1[\mathrm{M}+\mathrm{Na}]^{+}$.

$Q$-va. ${ }^{1} \mathrm{H}$ NMR chemical shifts: $\delta(\mathrm{ppm}) 12.23(1 \mathrm{H}, \mathrm{s}$, 5-OH), 7.32 (1H, d, H-2'), 7.27 (1H, d, H-6'), 6.92 (1H, d, H-5'), 6.48 (1H, d, H-8), 6.25 (1H, d, H-6), $2.65\left(2 \mathrm{H}, \mathrm{t}, \mathrm{CH}_{2}\right), 1.62$ $\left(2 \mathrm{H}, \mathrm{m}, \mathrm{CH}_{2}\right), 1.36\left(2 \mathrm{H}, \mathrm{m}, \mathrm{CH}_{2}\right), 0.90\left(3 \mathrm{H}, \mathrm{m}, \mathrm{CH}_{3}\right) .{ }^{13} \mathrm{C} \mathrm{NMR}$ chemical shifts: $\delta$ (ppm) $175.40(\mathrm{C}-4), 171.01(\mathrm{C}=\mathrm{O}), 165.22$ (C-7), 161.55 (C-9), 157.09 (C-5), 156.48 (C-2), 149.77 (C-4'), 145.97 (C-3'), 130.04 (C-3), 122.97 (C-1'), 120.07 (C-6'), 116.38 (C-5'), 115.49 (C-2'), 103.89 (C-10), 99.56 (C-6), 94.56 (C-8), $33.30\left(\mathrm{CH}_{2}\right), 26.85\left(\mathrm{CH}_{2}\right), 21.93\left(\mathrm{CH}_{2}\right), 14.04\left(\mathrm{CH}_{3}\right) . \mathrm{MS}(\mathrm{m} / \mathrm{z})$ : $409.1[\mathrm{M}+\mathrm{Na}]^{+}$.

In the ${ }^{13} \mathrm{C}$ NMR spectrum of quercetin, the chemical shift of C3 appears at 136.18 ppm. However, the chemical shift of C3 in the synthesized analogues moved upfield (130.06 ppm for Q-pr, $129.56 \mathrm{ppm}$ for Q-bu, and130.04 ppm for Q-va). Furthermore, the chemical shifts attributed to the ester carbonyl carbons of Q-pr, Q-bu and Q-va were identified at $171.78,170.48$ and $171.01 \mathrm{ppm}$, respectively. The ions at $\mathrm{m} / \mathrm{z}$
381.1, m/z 395.1 and $\mathrm{m} / \mathrm{z} 409.1$ in the MS spectra correspond to sodiated adducts $[\mathrm{M}+\mathrm{Na}]^{+}$for Q-pr, Q-bu and Q-va, respectively, consistent with the C3-substituted structures. This further confirmed the successful substitution of aliphatic acyls at $\mathrm{C} 3$.

Q-pr and Q-bu exhibited significantly improved water solubility (Table I). In particular, the water solubility of Q-pr was 8.2-fold higher compared with that of quercetin, while Q-bu exhibited a 4.7-fold higher water solubility. However, Q-va did not exhibit improved water solubility, as its water solubility was only $54 \%$ that of quercetin. These results suggested that the water solubility of quercetin analogues is affected by the carbon chain length at C3. Propionyl group has the shortest carbon chain, possessing a total of three carbons. It was shown to be the most effective in increasing water solubility. Butyryl group has one more methylene compared with propionyl group and a total of four carbons. It was still effective in increasing the water solubility of quercetin. However, the five-carbon valeroyl group failed to render the quercetin analogue more water soluble. This was probably because 3- and 4-carbon short aliphatic acyl chains were efficient in disrupting intermolecular packing of quercetin molecules, thus enabling solvent molecules to enter the intermolecular gap to solvate quercetin molecules more easily. A further increase in chain length to 5 carbons made the hydrophobic effect dominant, making quercetin substituted with valeroyl group more hydrophobic. Their $n$-octanol/water partition coefficients (Ps) were tested and three different methods were used to calculate $\log P$ (Table I). The lipophilicity of a compound is reflected by $\log P$. Acyl chain length increase resulted in stronger lipophilicity. The results indicate that the $\log P$ values of the acylated derivatives were higher than compared with of quercetin, and it increased with aliphatic acyl chain length with $\log P$ of Q-va being the highest.

Given that the acylation regioselectively occurred at $\mathrm{C} 3$ of quercetin and the bioactive hydroxyl groups remained unchanged, the biological activities of quercetin were expected to be maintained. The in vitro assay demonstrated that the three acylated derivatives were able to inhibit platelet aggregation induced by ADP, AA and PAF in a dose-dependent manner (Fig. 3). Q-pr and Q-bu were found to be more potent compared with quercetin. 

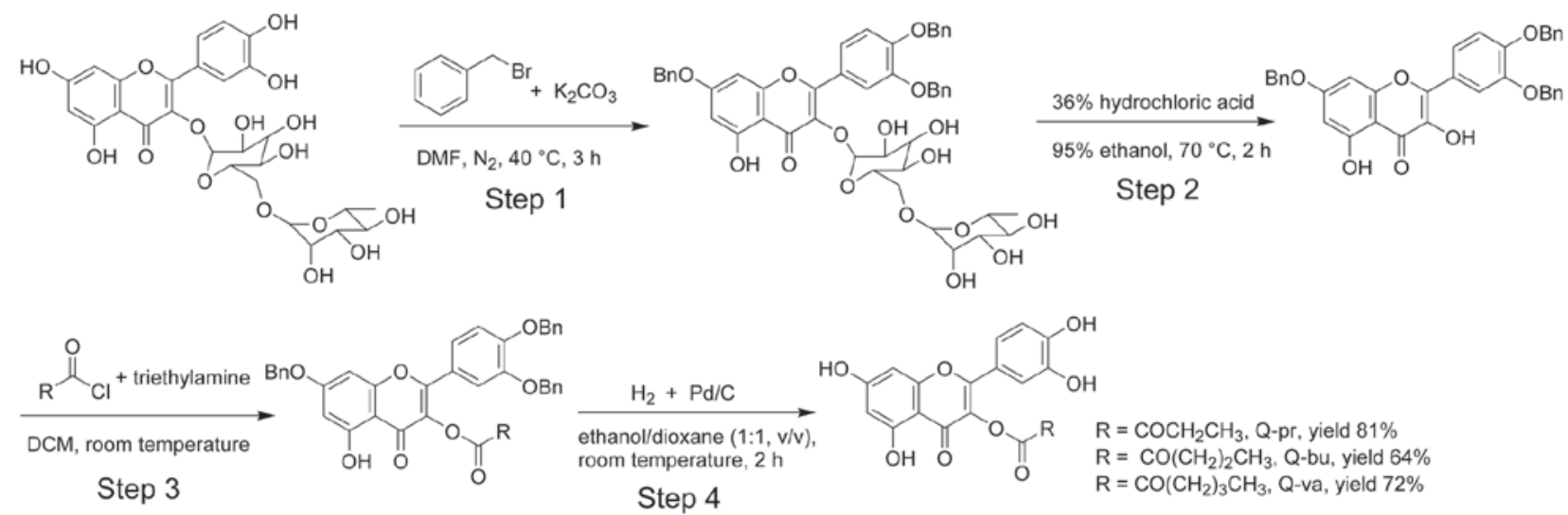

Figure 2. Synthetic approach to the synthesis of regioselectively acylated quercetin analogues. Q-pr, quercetin-3-O-propionate; Q-bu, quercetin-3-O-butyrate; Q-va, quercetin-3- $O$-valerate; DMF, $N, N$-dimethylformamide; DCM, dichloromethane; Pd/C, palladium on carbon.
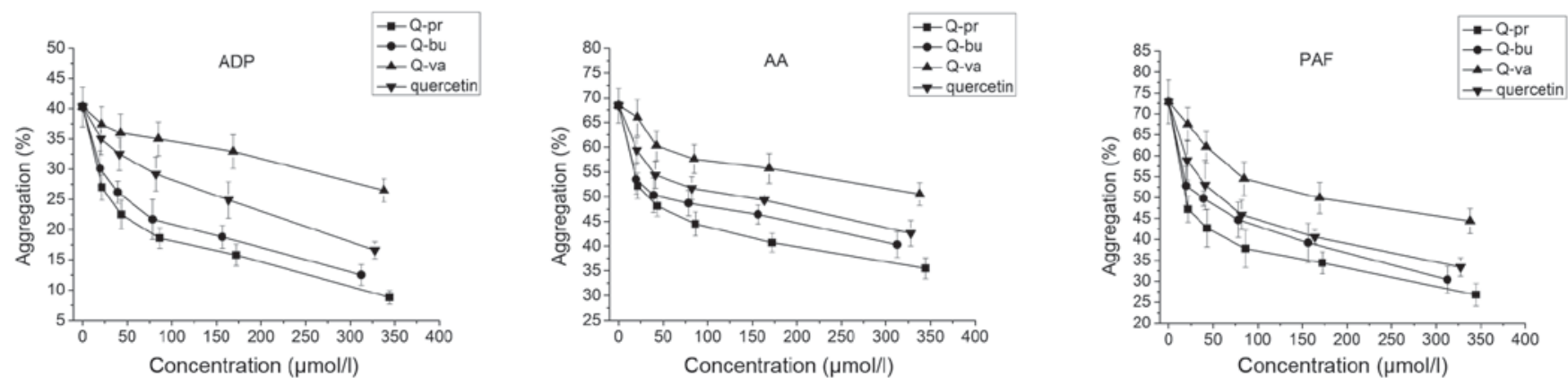

Figure 3. In vitro antiplatelet aggregation activities of quercetin, Q-pr, Q-bu, and Q-va. Q-pr, quercetin-3-O-propionate; Q-bu, quercetin-3-O-butyrate; $\mathrm{Q}$-va, quercetin-3- $O$-valerate; ADP, adenosine diphosphate; AA, arachidonic acid; PAF, platelet-activating factor.
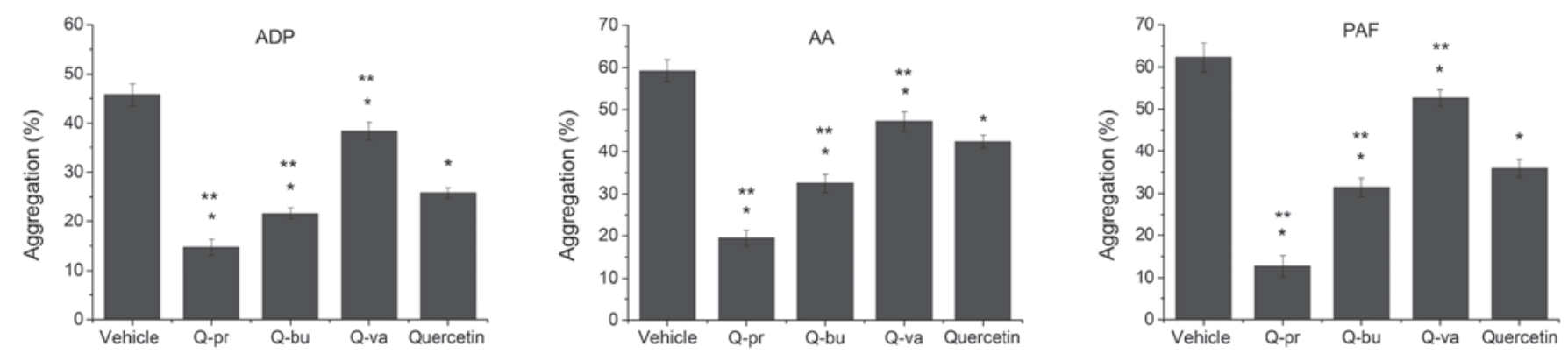

Figure 4. In vivo antiplatelet aggregation activities of quercetin, Q-pr, Q-bu, and Q-va. ${ }^{*} \mathrm{P} \leq 0.01$ vs. control. ${ }^{* *} \mathrm{P} \leq 0.01$ vs. quercetin. Q-pr, quercetin-3- $O$-propionate; $\mathrm{Q}$-bu, quercetin-3- $O$-butyrate; Q-va, quercetin-3- $O$-valerate; ADP, adenosine diphosphate; AA, arachidonic acid; PAF, platelet-activating factor.

Q-pr and Q-bu also displayed higher antiplatelet aggregation activities compared with quercetin in vivo (Fig. 4). In the ADP group, the AIRs of Q-pr and Q-bu were 67.8 and 52.7\%, respectively, which were significantly higher compared with that of quercetin (43.5\%). Although Q-va successfully inhibited platelet aggregation to a certain extent with an AIR of $16.0 \%$, it was significantly less potent compared with quercetin. The same order of drug activity of the four compounds was also observed in the AA and PAF groups.

In summary, regioselectively acylated quercetin analogues were successfully synthesized using a complete synthetic approach. The water solubility and lipophilicity of the resulting analogues were affected by the length of the aliphatic chains for acylation of the hydroxyl group located at $\mathrm{C} 3$ of quercetin. Aliphatic acyl donors containing three and four carbons were able to improve both the water solubility and the lipophilicity of the acylated analogues. As compared to quercetin, Q-pr and Q-bu exhibited a higher antiplatelet activity due to the higher water solubility and enhanced lipophilicity, whereas Q-va was less effective than quercetin due to its reduced water solubility, although its lipophilicity was the highest among the three analogues. Thus, an optimal acyl chain length is crucial for the quercetin analogues synthesized following this complete synthetic approach to be more effective. 


\section{Acknowledgements}

The authors would like to thank Jian Guo and Weijie Guo for providing assistance with the experiments. The present study was supported by the Shandong Natural Science Foundation (grant no.ZR2010HQ052), the Medical and Health Science and Technology Development Project of Shandong Province (grant nos. 2011QZ025 and 2014WSB27002), the Pharmaceutical Technology Development Project of Shandong Province (grant no. 2013-238) and the Chinese Key Technology Program (grant no. 2013GA740103).

\section{References}

1. Hertog MG, Hollman PC, Katan MB and Kromhout D: Intake of potentially anticarcinogenic flavonoids and their determinants in adults in the Netherlands. Nutr Cancer 20: 21-29, 1993.

2. Chen Y and Deuster P: Comparison of quercetin and dihydroquercetin: Antioxidant-independent actions on erythrocyte and platelet membrane. Chem Biol Interact 182: 7-12, 2009.

3. Fuentes E and Palomo I: Relationship between Platelet PPARs, cAMP levels, and P-Selectin expression: Antiplatelet activity of natural products. Evid Based Complement Alternat Med 2013: 861786, 2013.

4. Fuentes E and Palomo I: Antiplatelet effects of natural bioactive compounds by multiple targets: Food and drug interactions. J Funct Foods 6: 73-81, 2014

5. Mosawy S, Jackson DE, Woodman OL and Linden MD: Treatment with quercetin and 3',4'-dihydroxyflavonol inhibits platelet function and reduces thrombus formation in vivo. J Thromb Thrombolys 36: 50-57, 2013.

6. Navarro-Núñez L, Lozano ML, Martínez C, Vicente V and Rivera J: Effect of quercetin on platelet spreading on collagen and fibrinogen and on multiple platelet kinases. Fitoterapia 81: 75-80, 2010.

7. Navarro-Núñez L, Rivera J, Guerrero JA, Martínez C, Vicente V and Lozano ML: Differential effects of quercetin, apigenin and genistein on signalling pathways of protease-activated receptors PAR(1) and PAR(4) in platelets. Brit J Pharmacol 158: 1548-1556, 2009.

8. Oh WJ, Endale M, Park SC, Cho JY and Rhee MH: Dual roles of quercetin in platelets: Phosphoinositide-3-kinase and MAP kinases inhibition, and cAMP-dependent vasodilator-stimulated phosphoprotein stimulation. Evid Based Complement Alternat Med 2012: 485262, 2012.

9. Ferry DR, Smith A, Malkhandi J, Fyfe DW, deTakats PG, Anderson D, Baker J and Kerr DJ: Phase I clinical trial of the flavonoid quercetin: Pharmacokinetics and evidence for in vivo tyrosine kinase inhibition. Clin Cancer Res 2: 659-668, 1996.
10. Leonarduzzi G, Testa G, Sottero B, Gamba P and Poli G: Design and development of nanovehicle-based delivery systems for preventive or therapeutic supplementation with flavonoids. Curr Med Chem 17: 74-95, 2010.

11. Justino GC, Santos MR, Canário S, Borges C, Florêncio MH and Mira L: Plasma quercetin metabolites: Structure-antioxidant activity relationships. Arch Biochem Biophys 432: 109-121, 2004.

12. Wang H and Joseph JA: Structure-activity relationships of quercetin in antagonizing hydrogen peroxide-induced calcium dysregulation in PC12 cells. Free Radic Biol Med 27: 683-694, 1999.

13. Montenegro L, Carbone C, Maniscalco C, Lambusta D, Nicolosi G, Ventura CA and Puglisi G: In vitro evaluation of quercetin-3-O-acyl esters as topical prodrugs. Int J Pharm 336: 257-262, 2007.

14. Saija A, Tomaino A, Trombetta D, Pellegrino ML, Tita B, Messina C, Bonina FP, Rocco C, Nicolosi G and Castelli F: 'In vitro' antioxidant and photoprotective properties and interaction with model membranes of three new quercetin esters. Eur J Pharm Biopharm 56: 167-174, 2003.

15. Gatto MT, Falcocchio S, Grippa E, Mazzanti G, Battinelli L, Nicolosi G, Lambusta D and Saso L: Antimicrobial and anti-lipase activity of quercetin and its $\mathrm{C} 2-\mathrm{C} 163-\mathrm{O}$-acyl-esters. Bioorg Med Chem 10: 269-272, 2002.

16. Kumar V, Jahan F, Mahajan RV and Saxena RK: Efficient regioselective acylation of quercetin using Rhizopus oryzae lipase and its potential as antioxidant. Bioresour Technol 218: 1246-1248, 2016.

17. Sardone L, Pignataro B, Castelli F, Sarpietro MG, Nicolosi G and Marletta G: Temperature and pressure dependence of quercetin-3-O-palmitate interaction with a model phospholipid membrane: Film balance and scanning probe microscopy study. J Colloid Interface Sci 271: 329-335, 2004.

18. Yamauchi K, Mitsunaga T, Inagaki M and Suzuki T: Synthesized quercetin derivatives stimulate melanogenesis in B16 melanoma cells by influencing the expression of melanin biosynthesis proteins MITF and p38 MAPK. Bioorg Med Chem 22: 3331-3340, 2014.

19. Wuts PGM and Greene TW: Protection for Phenols and Catechols. In: Greene's Protective Groups in Organic Synthesis. John Wiley \& Sons, New York, pp367-430, 2006.

20. Bonina FP, Montenegro L, De Capraris P, Bousquet E and Tirendi S: 1-Alkylazacycloalkan-2-one esters as prodrugs of indomethacin for improved delivery through human skin. Int J Pharm 77: 21-29, 1991.

21. Braumann T: Determination of hydrophobic parameters by reversed-phase liquid chromatography: Theory, experimental-techniques, and application in studies on quantitative structure-activity-relationships. J Chromatogr 373: 191-225, 1986.

22. Mosawy S, Jackson DE, Woodman OL and Linden MD: Treatment with quercetin and 3',4'-dihydroxyflavonol inhibits platelet function and reduces thrombus formation in vivo. J Thromb Thrombolys 36: 50-57,2013. 